\title{
Research Paper: \\ Blockade of Hypoxia: The Impact on Tumor Growth in an Experimental Tumor Model
}

\author{
Nasim Kheshtchin ${ }^{1}$, Samaneh Arab $^{2,3}$ (D), Jamshid Hadjati ${ }^{1 *}$ (D) \\ 1. Department of Medical Immunology, School of Medicine, Tehran University of Medical Sciences, Tehran, Iran. \\ 2. Department of Tissue Engineering and Applied Cell Sciences, School of Medicine, Semnan University of Medical Sciences, Semnan, Iran. \\ 3. Neural Stem Cells Research Center, Semnan University of Medical Sciences, Semnan, Iran.
}

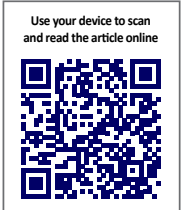

Citation Kheshtchin N, Arab S, Hadjati J. Blockade of Hypoxia: The Impact on Tumor Growth in an Experimental Tumor Model. Immunoregulation. 2019; 2(1):35-40. http://dx.doi.org/10.32598/Immunoregulation.1.3.153

dolo http://dx.doi.org/10.32598/Immunoregulation.1.3.153

(c) (i) (\$)

Article info:

Received: 29 Feb 2018

Accepted: 05 Jul 2018

Available Online: 01 July 2019

\section{Keywords:}

Cancer, Hypoxia-

Inducible Factor- $1 \alpha$ (HIF-

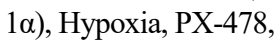

Foxp3, VEGF

\begin{abstract}
A B S T R A C T
Background: Tumor microenvironment is an active factor participating in immunoregulation, thereby preventing immunosurveillance and limiting the efficacy of anticancer therapies. Hypoxia as a major characteristic of solid tumors causes the expression of Hypoxia-Inducible Factor- $1 \alpha$ (HIF$1 \alpha$ ). This is a transcription factor that mediates hypoxic responses of tumor cells and involves in the expression of tumor immunosuppression-related genes.
\end{abstract}

Materials and Methods: In this study, we used a mouse 4T1 breast cancer model.

Results: Our obtained data revealed that in vivo administration of PX-478, an inhibitor of oxygen sensitive HIF-1 $\alpha$, reduced the expression of Forkhead box P3 (Foxp3) transcript, a molecule that is directly controlled by HIF-1. The level of vascular endothelial growth factor, another gene controlled by HIF-1, remained unchanged. The observed results were in correlation with delayed tumor growth in tumor-bearing mice.

Conclusion: Our findings indicate that the reduction in Foxp3 expression through HIF-1 $\alpha$ inhibition using PX-478 may contribute to tumor regression.

\footnotetext{
* Corresponding Author:

Jamshid Hadjati, PhD.

Address: Department of Medical Immunology, School of Medicine, Tehran University of Medical Sciences, Tehran, Iran.

Phone: +98 (21) 64053268

E-mail: hajatij@sina.tums.ac.ir
} 


\section{Introduction}

$\mathbf{H}$

ypoxia, a common characteristic of all solid tumors, provokes mechanisms involved in suppressing antitumor immunity and protects cancerous tissues [1-5]. The immunoregulatory effect of hypoxia is elicited by Hypoxia-Inducible Factor (HIF). HIF mediates adaptive transcriptional responses to hypoxia, involved in different cell biology aspects, including cell survival, glucose metabolism, angiogenesis, and invasion [6, 7]. HIF is a heterodimer molecule consisting of constitutively expressed beta (HIF-1 $\beta$ ) subunit and one of the three oxygen-regulated alpha subunits (HIF-1 $\alpha$, HIF-2 $\alpha$, or HIF- $3 \alpha$ ). In the absence of oxygen, alpha subunits are post-translationally stabilized and heterodimerized with HIF-1 $\beta$, then be translocated into the nucleus and transactivate its target genes [8].

Hypoxia plays a critical role in immune regulation and resistance to therapy. Thus, targeting HIF-1 activity could be a potential immunotherapeutic approach for cancer therapy. Suppressing HIF-1 in animal models decreases tumorigenesis, and increases survival rate [9]. A growing number of medications have been introduced as anticancer agents that are HIF-1inhibitors [8, 10]. PX-478, [S-2-amino-3-(4V-N,N,-bis[2-chloroethyl]amino)-phenyl propionic acid N-oxide dihydrochloride], is a small molecule that suppresses hypoxic and normoxic translations of HIF-1 $\alpha$, as well as HIF-1 $\alpha$ transcriptional activity and Vascular Endothelial Growth Factor (VEGF) expression under hypoxic conditions, in various cancer cell lines [11, 12]. Moreover, hypoxia promotes Foxp3 expression and regulatory T-cell function through direct transcriptional activation of Foxp3 mRNA by HIF-1 $\alpha$ [13].

Given the impact of hypoxic stress on angiogenesis, tumor progression and immune tolerance, hypoxia attracted particular attention in tumor immune biology. The present study investigated the effect of HIF- $1 \alpha$ inhibition using PX478 on tumor growth in the mouse model of cancer, as well as its potential effect on VEGF and Foxp3 expression.

\section{Materials and Methods}

\section{Mice and cell lines}

Six- to 8-week-old BALB/c and C57BL/6 female mice were obtained from the Laboratory Animal Center, Pasteur Institute of Iran. Animal protocols were approved by the Institutional Animal Care and Use Committee of Tehran University of Medical Sciences. The 4T1 carcinoma and F10 melanoma cell lines which are of BALB/c and $\mathrm{C} 57 \mathrm{BL} / 6$ origin, respectively, were cultured in complete media RPMI-1640 (Biosera, UK) supplemented with 100 $\mathrm{U} / \mathrm{mL}$ penicillin, $100 \mu \mathrm{g} / \mathrm{mL}$ streptomycin, $10 \mathrm{mmol} / \mathrm{L} \mathrm{L}$ glutamine (Biosera, UK), as well as 10\% heat-inactivated FBS (Gibco, Grand Island, USA), in a $5 \% \mathrm{CO}_{2}$ humidified incubator at $37^{\circ} \mathrm{C}$. Tumors were created by subcutaneously injecting $7 \times 1054 \mathrm{~T} 1$ or $5 \times 105 \mathrm{~F} 10$ tumor cells into the right flank of syngeneic mice, with tumor size (in $\mathrm{mm}^{2}$ ), assessed every 2 days thereafter.

\section{PX-478 treatment}

HIF-1 $\alpha$ inhibitor, PX-478, was purchased from MedKoo Biosciences. For in vivo use, tumor-bearing mice were Intraperitoneally (IP) administered 20, 40 or $60 \mathrm{mg} / \mathrm{kg}$ PX478 or normal saline in a total volume of $200 \mu \mathrm{L}, 3$ times a week for one week, beginning after tumors were palpable with an approximate size of $\sim 25 \mathrm{~mm}^{2}$ in the area.

\section{RNA isolation and real-time quantitative PCR}

RNA was extracted from frozen tissues with Hybrid-R RNA purification kit (GeneAll Biotechnology, Korea). One microgram of RNA was reverse transcribed into complementary DNA (cDNA), using a QuantiTect Reverse Transcription kit (Qiagen). cDNAs were quantified by real-time PCR using an SYBR Green Real-time PCR master mix (Primer design, UK), on an ABI 7500 detection system (Applied Biosystems, United States). The relative mRNA levels were determined using the $\Delta \mathrm{Ct}$ method. The values were expressed relative to endogenous $\beta$-actin. The following PCR primers were used: HIF-1 $\alpha$ forward, 5' AGCTTCTGTTATGAGGCTCACC-3'; HIF-1 $\alpha$ reverse, 5'- TGACTTGATGTTCATCGTCCTC-3' [14]; VEGF forward, 5'- GCGGAGAAAGCATTTGTTTG-3'; VEGF reverse, 5'-TCTTTCCGGTGAGAGGTCTG-3'; Foxp3 forward, 5'-GCAGGGCAGCTAGGTATCTGTAG-3'; Foxp3 reverse, 5'-TCGGAGATCCCCTTTGTCTTATC-3'; $\beta$-actin forward, 5'-GGTCATCACTATTGGCAACG-3'; and $\beta$-actin reverse, 5'-ACGGATGTCAACGTCACACT-3'.

\section{Statistical analysis}

Between-group comparisons were performed using 1-way Analysis of Variance (ANOVA), and Tukey's test. GraphPad Prism was used for graphs and statistical analysis. $\mathrm{P}$ values less than 0.05 were considered as significance.

\section{Results}

PX-478 administration interferes with HIF-1a and Foxp3 expression

PX-478 is a HIF-1 $\alpha$ inhibitor currently being evaluated in phase I/II clinical trials. Previous studies indicated that 
a)

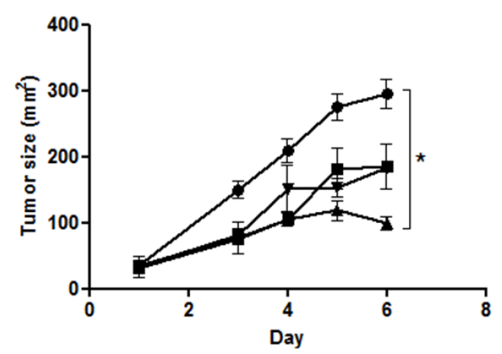

b)

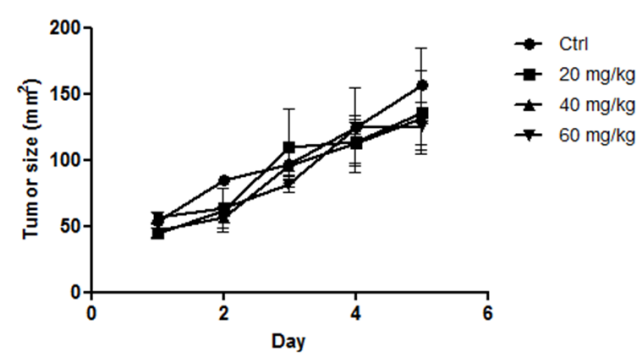

IMMUNoRecLLATION

Figure 1. Effect of PX-478 treatment on the expression of HIF-1a and its downstream genes Tumor-bearing mice were left untreated or they were given PX-478 (IP at 20, 40, or $60 \mathrm{mg} / \mathrm{kg}$ every other day for a week). Tu-
mors were excised one day after the final injection. Then, HIF-1a mRNA in both models, as well as VEGF and Foxp3 mRNA in breast carcinoma model was measured in tumors by real-time RT-PCR, calculated relative to housekeeping gene $\beta$-actin. Data were expressed as the mean \pm SEM for the three mice per group. ${ }^{*}<0.05$ by 1 -way ANOVA.

PX-478 administration inhibits HIF-1 $\alpha$ expression and its downstream target gene, VEGF. The direct effect of HIF-1 $\alpha$ on the induction of Foxp3 has also been indicated. To determine whether PX-478 involves VEGF and Foxp3 expression, PX-478 was IP administered in palpable tumors, at dosages of 20,40 , or $60 \mathrm{mg} / \mathrm{kg}$ every other day for a week. Treated animals were sacrificed 1 day after receiving the last dose of the drug, and tumors were excised. Then, the transcript levels of HIF-1 $\alpha$, FOXP3, and VEGF were measured via real-time RT-PCR.
As shown in Figure 1, HIF-1 $\alpha$ transcription significantly decreased in tumors obtained from 4T1 tumor-bearing mice that received $40 \mathrm{mg} / \mathrm{kg}$ PX-478. However, in the case of F10 tumors, HIF-1 $\alpha$ expression, while not at a significant level, indicated a decreased expression at the dosages of 40 and $60 \mathrm{mg} / \mathrm{kg}$. These studies revealed that HIF-1 $\alpha$ blockade diminished the tumoral expression of Foxp3 when the inhibitor was administrated at $40 \mathrm{mg} / \mathrm{kg}$ in breast cancer models. However, VEGF expression evaluated at mRNA level suggested no remarkable change in tumors.

a)
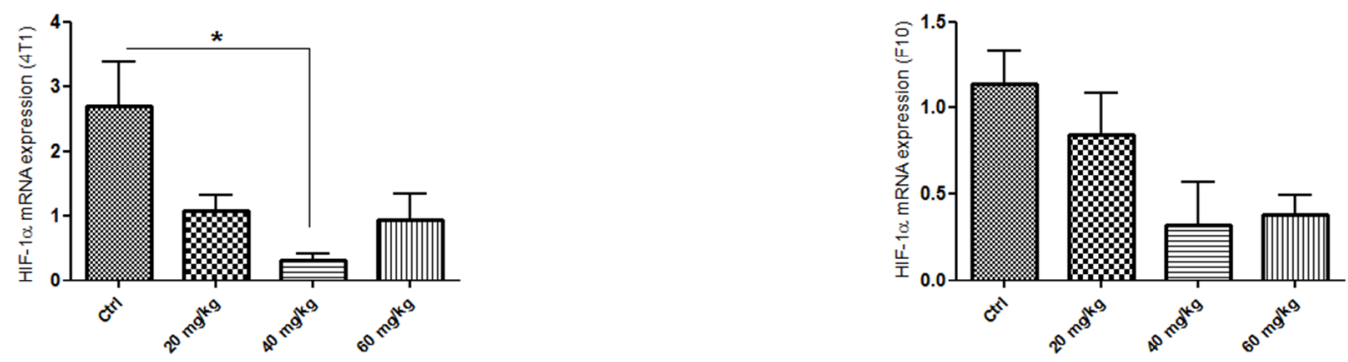

b)
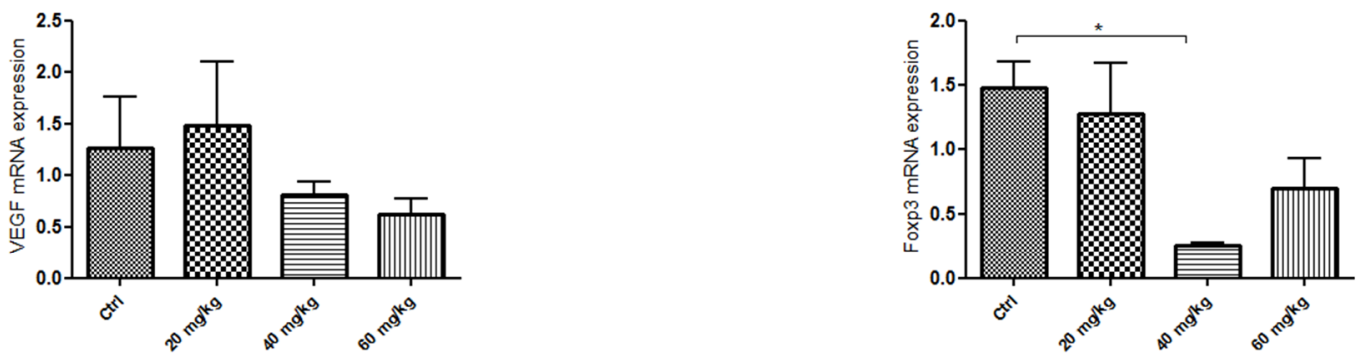

Figure 2. Delay of tumor growth in mice treated with PX-478

IMnUNoRegulation

Tumor size (mean $\pm \mathrm{SEM}$ ) of mice in each treatment modality reported in $\mathrm{mm}^{2}$ followed till the end of the experiment (a. 4T1; and $b$. F10 model). ${ }^{*}<0.05$ (ANOVA, $n=3$ ). 
PX-478 administration at $40 \mathrm{mg} / \mathrm{kg}$ promotes tumor regression in $\mathrm{BALB} / \mathrm{c}$ mice

The effect of different dosages of PX-478 on tumor progression was investigated using subcutaneously implanted 4T1 and F10 tumor cell grafts in syngeneic animals. As shown in Figure 2, untreated tumors in 4T1 tumor bearingmice displayed rapidly progressive growth, whereas tumors in animals treated with 20 and $60 \mathrm{mg} / \mathrm{kg}$ PX-478 grew more slowly. The growth of tumors in mice treated with $40 \mathrm{mg} /$ kg PX-478 significantly decreased, compared with the untreated group. PX-478 treatment in mice bearing F10 tumors indicated no significant effects on tumor growth.

\section{Discussion}

The hypoxia of solid tumors as a tumor microenvironment hallmark is strongly associated with malignant phenotypes and therapy resistance [15]. HIF-1, the master regulator of the cellular response to hypoxia, is targeted for cancer therapy. Due to the critical role of HIF-1 in cancer progression, there is interest in the discovery of medications that target this molecule $[6,16,17]$. PX-478 is a specific agent that suppresses HIF-1 $\alpha$ levels under normoxic and hypoxic conditions in various cancer cell lines $[11,12]$.

During hypoxia, multiple anti-inflammatory mechanisms might be elicited via HIF-dependent transactivation, like VEGF induction that plays an important role in tumor angiogenesis and immune escape $[18,19]$. Another example is the induction of extracellular adenosine pathway that contributes to Regulatory $\mathrm{T}$ cells (Treg) differentiation and function [20-22]. Another mechanism is the enhanced suppressing effect of tumor Myeloid-Derived Suppressor Cells (MDSCs), that involves HIF-1 $\alpha$-mediated induction of PDL-1 on these cells [23]. Moreover, upon TCR activation, CD4+ T cells upregulate Foxp3 expression in a HIF- $1 \alpha$ and Transforming Growth Factor (TGF)- $\beta$-dependent manner; thereby differentiate to Tregs. The differentiated Tregs demonstrate defects in their inhibitory functions in the absence of HIF-1 $\alpha$ [13].

The present study explored the effects of HIF-1 $\alpha$ blockade by PX-478 on tumor growth, as well as the tumor expression of VEGF and Foxp3 genes. When used at $40 \mathrm{mg} / \mathrm{kg}$, PX478 was particularly effective in the breast cancer model. This is because it led to a marked reduction in tumor growth in correlation with a significant decrease in HIF-1 $\alpha$ mRNA. However, with no significant effect on HIF-1 $\alpha$ expression, the drug was not effective on the F10 tumor model. This finding indicates that tumors induced by F10 cells might be resistant to HIF-1 inhibition, or the selected range of drug concentration is not effective on the tumors induced by this cell line. Prior research also reported that the effectiveness of PX-478 is positively correlated with HIF-1 expression in tumors [12]. The microenvironment of the melanoma or the administered drug dose might influence the effectiveness of PX-478 on HIF-1 $\alpha$ inhibition. Moreover, original studies were performed using higher dosages of this drug.

We also investigated the expression of VEGF and Foxp3 genes that are directly controlled by HIF-1 $\alpha$. The treatment of BALB/c mice with $40 \mathrm{mg} / \mathrm{kg}$ PX-478 significantly reduced Foxp3 expression. However, an unexpected observation was that PX-478 indicated no significant effect on VEGF expression in tumor tissues obtained from the treated mice. Various studies also emphasized the role of HIF-1 $\alpha$ in tumor progression and angiogenesis by macrophages. Doedens et al. indicated that VEGF is a HIF-1 $\alpha$ regulated gene [24].

The targeted deletion of HIF-1 $\alpha$ in myeloid cells failed to change VEGF-A levels or tumor vasculature. The tumors, however, exhibited reduced progression attributed to their escape from immune suppression. The reduced expression of tumoral Foxp3 observed in the present study might be an indication of impairments in the number of Treg cells or their function. However, another study reported that HIF- $1 \alpha$ promotes Foxp3 degradation in the proteasome [25], emphasizing the determinant role of different microenvironments in the effects of HIF-1 on Foxp3, and Treg function. The precise effect of hypoxia on Tregs in the tumor microenvironment remains largely unexplored.

Our study revealed that HIF-1 $\alpha$ inhibition using PX-478 is highly effective on the mouse models of breast cancer. PX-478 treatment significantly reduced Foxp3 expression and decreased tumor growth in a dose-dependent manner in the breast carcinoma model, when given as monotherapy for just 3 days.

\section{Ethical Considerations}

\section{Compliance with ethical guidelines}

All ethical principles were considered in this article. The participants were informed about the purpose of the research and its implementation stages; they were also assured about the confidentiality of their information; Moreover, They were allowed to leave the study whenever they wish, and if desired, the results of the research would be available to them.

\section{Funding}

This work was supported by grant No. 21191 by Tehran University of Medical Sciences. 


\section{Authors contributions}

All authors contributed in preparing this article.

Conflict of interest

The authors declared no conflict of interest.

\section{References}

[1] Facciabene A, Peng X, Hagemann IS, Balint K, Barchetti A Wang LP, et al. Tumor hypoxia promotes tolerance and angiogenesis via CCL28 and T(reg) cells. Nature. 2011; 475(7355):226-30. [DOI:10.1038/nature10169] [PMID]

[2] Sitkovsky M, Lukashev D. Regulation of immune cells by local-tissue oxygen tension: HIFla and adenosine receptors. Nature Reviews Immunology. 2005; 5(9):712-21. [DOI:10.1038/nri1685] [PMID]

[3] Noman MZ, Benlalam H, Hasmim M, Chouaib S. Cytotoxic T cells-stroma interactions. Bull Cancer. 2011; 98(2):E19-24. [PMID]

[4] Noman MZ, Janji B, Kaminska B, Van Moer K, Pierson S, Przanowski $\mathrm{P}$, et al. Blocking hypoxia-induced autophagy in tumors restores cytotoxic T-cell activity and promotes regression. Cancer Research. 2011; 71(18):5976-86. [DOI:10.1158/0008-5472.CAN-11-1094] [PMID]

[5] Noman MZ, Messai Y, Carre T, Akalay I, Meron M, Janji B, et al. Microenvironmental hypoxia orchestrating the cell stroma cross talk, tumor progression and antitumor response. Critical Reviews ${ }^{\mathrm{TM}}$ in Immunology. 2011; 31(5):357-77. [DOI:10.1615/CritRevImmunol.v31.i5.10] [PMID]

[6] Semenza GL. Targeting HIF-1 for cancer therapy. Nature Reviews Cancer. 2003; 3(10):721-32. [DOI:10.1038/nrc1187] [PMID]

[7] Shi YH, Fang WG. Hypoxia-Inducible Factor-1 in tumor angiogenesis. World Journal of Gastroenterology. 2004; 10(8):1082-7. [DOI:10.3748/wjg.v10.i8.1082] [PMCID]

[8] Semenza GL. Defining the role of Hypoxia-Inducible Factor 1 in cancer biology and therapeutics. Oncogene. 2010; 29(5):625-34. [DOI:10.1038/onc.2009.441] [PMID] [PMCID]

[9] Kim JwG, Ping D, Chi V. Effects of hypoxia on tumor metabolism. Cancer and Metastasis Reviews. 2007; 26(2):291-8. [DOI:10.1007/s10555-007-9060-4] [PMID]

[10] Wilson WR, Hay MP. Targeting hypoxia in cancer therapy. Nature Reviews Cancer. 2011; 11(6):393-410. [DOI:10.1038/ nrc3064] [PMID]

[11] Koh MY, Spivak Kroizman T, Venturini S, Welsh S, Williams RR, Kirkpatrick DL, et al. Molecular mechanisms for the activity of PX-478, an antitumor inhibitor of the Hypoxia-Inducible Factor-1alpha. Molecular Cancer Therapeutics. 2008; 7(1):90100. [DOI:10.1158/1535-7163.MCT-07-0463] [PMID]

[12] Welsh S, Williams R, Kirkpatrick L, Paine Murrieta G, Powis G. Antitumor activity and pharmacodynamic properties of PX-478, an inhibitor of Hypoxia-Inducible Factor-1alpha. Molecular Cancer Therapeutics. 2004; 3(3):233-44. [PMID]
[13] Clambey ET, McNamee EN, Westrich JA, Glover LE, Campbell EL, Jedlicka P. Hypoxia-Inducible Factor-1 alpha-dependent induction of FoxP3 drives regulatory T-cell abundance and function during inflammatory hypoxia of the mucosa. National Academy of Sciences of the United States of America. 2012; 109(41):E2784-93. [DOI:10.1073/ pnas.1202366109] [PMID] [PMCID]

[14] Shi LZ, Wang R, Huang G, Vogel P, Neale G, Green DR, Chi H. HIF1a-dependent glycolytic pathway orchestrates a metabolic checkpoint for the differentiation of TH17 and Treg cells. Journal of Experimental Medicine. 2011; 208(7):136776. [DOI:10.1084/jem.20110278] [PMCID]

[15] Vergis R, Corbishley CM, Norman AR, Bartlett J, Jhavar S, Borre $\mathrm{M}$, et al. Intrinsic markers of tumor hypoxia and angiogenesis in localised prostate cancer and outcome of radical treatment: A retrospective analysis of two randomised radiotherapy trials and one surgical cohort study. The Lancet Oncology. 2008; 9(4):342-51. [DOI:10.1016/S14702045(08)70076-7]

[16] Dewhirst MW, Cao Y, Moeller B. Cycling hypoxia and free radicals regulate angiogenesis and radiotherapy response. Nature Reviews Cancer. 2008; 8(6):425-37. [DOI:10.1038/ nrc2438] [PMCID]

[17] Melillo G. Targeting hypoxia cell signaling for cancer therapy. Cancer and Metastasis Reviews. 2007; 26(2):341-52. [DOI:10.1007/s10555-007-9059-x]

[18] Takenaga K. Angiogenic signaling aberrantly induced by tumor hypoxia. Frontiers in Bioscience. 2011; 16:31-48. [DOI:10.2741/3674]

[19] Noman MZ, Buart S, Van Pelt J, Richon C, Hasmim M, Leleu N, et al. The cooperative induction of Hypoxia-Inducible Factor-1 alpha and STAT3 during hypoxia induced an impairment of tumor susceptibility to CTL-mediated cell lysis. The Journal of Immunology. 2009; 182(6):3510-21. [DOI:10.4049/jimmunol.0800854]

[20] Eltzschig HK, Ibla JC, Furuta GT, Leonard MO, Jacobson $\mathrm{KA}$, Enjyoji K, et al. Coordinated adenine nucleotide phosphohydrolysis and nucleoside signaling in posthypoxic endothelium: Role of ectonucleotidases and adenosine A2B receptors. The Journal of Experimental Medicine. 2003; 198(5):783-96. [DOI:10.1084/jem.20030891] [PMCID]

[21] Deaglio S, Dwyer KM, Gao W, Friedman D, Usheva A, Erat A, et al. Adenosine generation catalyzed by CD39 and CD73 expressed on regulatory $\mathrm{T}$ cells mediates immune suppression. The Journal of Experimental Medicine. 2007; 204(6):1257-65. [DOI:10.1084/jem.20062512] [PMCID]

[22] Zarek P, Huang CT, Lutz ER, Kowalski J, Horton MR, Linden J, et al. A2A receptor signaling promotes peripheral tolerance by inducing T-cell anergy and the generation of adaptive regulatory T cells. Blood. 2008; 111(1):251-9. [DOI:10.1182/blood-2007-03-081646] [PMCID]

[23] Noman MZ, Desantis G, Janji B, Hasmim M, Karray S, Dessen $\mathrm{P}$, et al. PD-L1 is a novel direct target of HIF-1, and its blockade under hypoxia enhanced MDSC-mediated T cell activation. The Journal of Experimental Medicine. 2014; 211(5):781-90. [DOI:10.1084/jem.20131916] [PMCID]

[24] Doedens AL, Stockmann C, Rubinstein MP. Macrophage expression of Hypoxia-Inducible Factor-1 alpha suppresses $\mathrm{T}$-cell function and promotes tumor progression. Cancer Re- 
search. 2010; 70(19):7465-75. [DOI:10.1158/0008-5472.CAN10-1439] [PMCID]

[25] Dang EV, Barbi J, Yang HY, Jinasena D, Yu H, Zheng Y, et al. Control of TH17/treg balance by Hypoxia-Inducible Factor 1. Cell. 2011; 146(5):772-84. [DOI:10.1016/j.cell.2011.07.033] [PMCID] 